\title{
Análogos de insulina: en búsqueda del reemplazo fisiológico
}

\author{
Francisca Eyzaguirre $C^{1, a}$, Ethel Codner². \\ Insulin analogues: searching for a \\ physiological replacement
}

Insulin analogues, developed by molecular engineering, have structural changes in the A and B insulin chains. These modifications change their action profile, rendering insulin replacement closer to physiology. Rapid acting analogues like lispro, aspart and glulisine, are absorbed rapidly from the subcutaneous tissue to the circulation. In addition, two long acting insulin analogues have been developed: glargine and detemir. The combination of a long acting insulin, to maintain baseline levels, and multiple daily doses of a rapid acting analogue are the mainstay of basal-bolus therapy. Multiples studies have compared human insulin (NPH and regular) with insulin analogues in patients with type 1 or 2 diabetes mellitus, showing an improvement in the metabolic control, fewer hypoglycemic events and better quality of life. In summary, insulin analogues offer new therapeutic options and allow an individualized intensive treatment (Rev Méd Chile 2006; 134: 239-50).

(Key w ords: Diabetes mellitus, insulin dependent; Insulin; Insulin, long acting; Pro insulin)

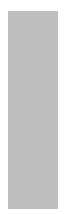

Recibido el 16 de marzo, 2005. Aceptado el 26 de mayo, 2005.

Trabajo financiado parcialmente por el proyecto FONDECYT \#1050452.

${ }^{1}$ Hospital Clínico San Borja Arriarán, Universidad de Chile, Campus Centro. ${ }^{2}$ Instituto de Investigaciones Materno Infantil (I.D.I.M.I.), Facultad de Medicina, Universidad de Chile, Santiago, Chile y Clínica Alemana, Santiago, Chile.

aBecada Pediatría

$E^{\mathrm{n}}$ los últimos años se han producido avances importantes en el tratamiento de la diabetes mellitus (DM). Inicialmente se publicaron dos grandes estudios prospectivos, el DCCT en DM tipo 1 (DM1) y el UKPDS en DM tipo 2 (DM2) ${ }^{1,2}$, quienes demostraron que la disminución de la glicemia reduce significativamente la aparición de

Correspondencia a: Dra. Ethel Codner D. IDIMI, Universidad de Chile. Casilla 226-3, Santiago, Chile. Teléfono: 562-4248280. Fax: 562-424-7240. E mail: ecodner@med.uchile.cl complicaciones microvasculares. Otro gran avance ha sido el desarrollo de los análogos de insulina. Con ellos se ha buscado un reemplazo insulínico más semejante a la secreción fisiológica, mediante esquemas intensificados ${ }^{3}$.

Los grandes beneficios que otorgan esquemas intensificados en el control de la DM motivaron al Ministerio de Salud de Chile a incluir a la DM1 y DM2 entre las patologías con garantías explícitas (AUGE) ${ }^{4}$. Asimismo, existen reportes chilenos que demuestran que es posible instaurar esquemas con múltiples dosis en el sistema público de salud 5,6 . 


\section{SECRECIÓN DE INSULINA DURANTE EL PERÍODO POSTPRANDIAL EN SUJETOS SANOS}

En sujetos sanos, inmediatamente después de ingerir alimentos, se secreta insulina desde las células $ß$ a la circulación portal, alcanzando insulinemias elevadas, con niveles máximos de hasta $80 \mathrm{uU} / \mathrm{ml}$ a los $30 \mathrm{~min}^{7}$. La insulina secretada se unirá a las células del tejido adiposo, hígado y músculo, estimulando la entrada de nutrientes a la célula, y evitando así un ascenso excesivo de la glicemia postprandial. Estos niveles retornan a niveles basales a las 2-3 h de la ingesta $^{8}$. La dificultad en imitar la secreción fisiológica en un paciente con DM1, está dada por la necesidad de un ascenso rápido de insulina postprandial paralelo al aumento de la glicemia y posteriormente la mantención de niveles basales de insulina hasta la siguiente comida.

\section{REEMPLAZO DE INSULINA DURANTE EL PERÍODO} POSTPRANDIAL

Insulina cristalina (IC). Hasta la década 1980-89 era la única insulina prandial disponible. Sus cristales corresponden a hexámeros de insulina producidos por agregados de la hormona unidos entre sí a través de la cadena B. Normalmente, la porción terminal de la cadena $\mathrm{B}$ tiene una disposición $ß$ plegada que tiende a asociarse con otras moléculas en forma antiparalela, no covalente, pero estable. Estos dímeros, en presencia de zinc, se asocian en grupos de tres, originando hexámeros, que en el tejido subcutáneo se disocian lentamente a dímeros y monómeros, retardando su paso a la circulación sistémica ${ }^{9}$.

La absorción lenta de IC determina una tardanza en su paso a la circulación con respecto a la máxima absorción de glucosa desde el tubo digestivo. Este es el fundamento para indicar la inyección media hora antes de comer, acción que generalmente no se cumple estrictamente ${ }^{10}$.

La IC no es una verdadera insulina prandial, porque los niveles máximos alcanzados son inferiores a los de un individuo sano ${ }^{7,11}$, determinando frecuentemente hiperglicemia postprandial. Asimismo, mantiene niveles elevados por 5-6 $\mathrm{h}^{12,13}$, comportándose durante este período como una insulina basal (Figura 1). Este hecho aumenta el riesgo de hipoglicemia y, por ende, obliga a ingerir una colación tardía ${ }^{7}$.

Análogos de insulina de uso prandial (Tablas 1 y 2 y Figuras 1 y 2). Son moléculas que difieren de la insulina en la secuencia de aminoácidos, pero son capaces de unirse a su receptor y actuar como ella ${ }^{9}$. Estos cambios aminoacídicos modifican su velocidad de absorción desde el subcutáneo y le otorgan ventajas farmacológicas por asemejarse más a la secreción de insulina durante el período de ayuno ( (basal») y prandial ${ }^{9,14}$.

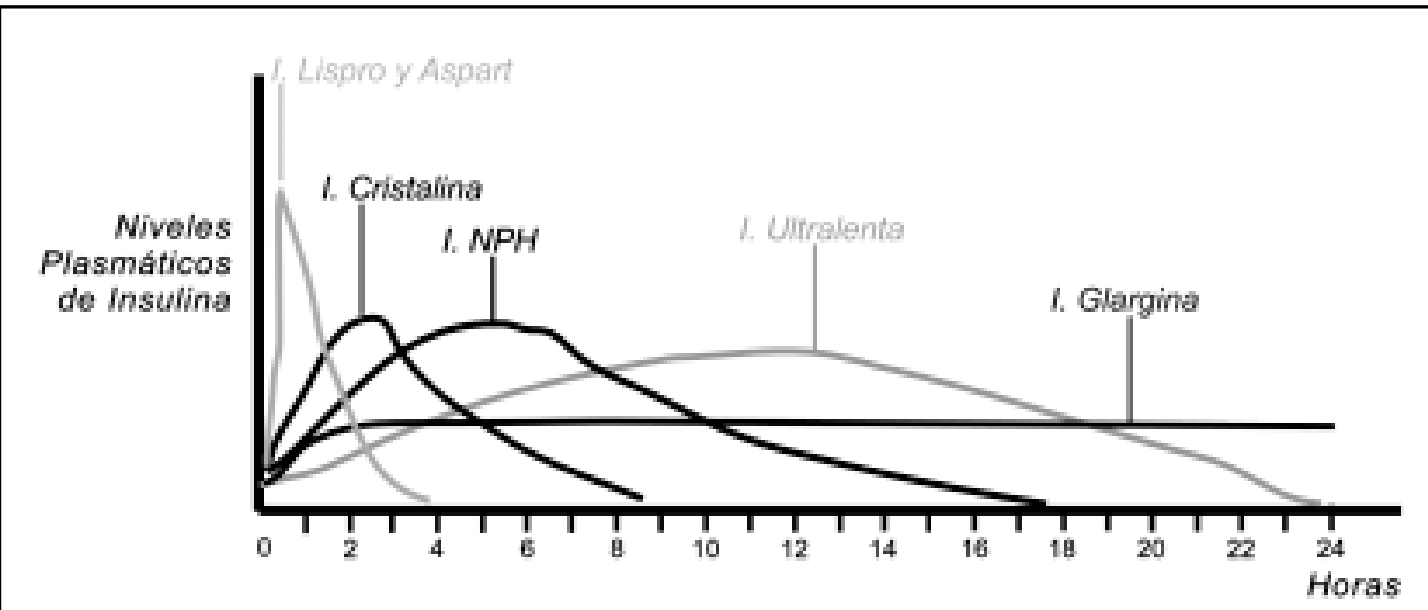

Figura 1. Esquema de los niveles plasmáticos de insulina logrados después de una inyección subcutánea. 


\section{Tabla 1. Comparación estructural y funcional de los análogos de insulina}

\begin{tabular}{|c|c|c|c|c|}
\hline & Cambio estructural & $\begin{array}{c}\text { Tiempo de } \\
\text { inicio de acción }\end{array}$ & $\begin{array}{l}\text { Tiempo en } \\
\text { alcanzar el peak } \\
\text { (minutos) }\end{array}$ & $\begin{array}{l}\text { Duración } \\
\text { de la acción } \\
\text { (horas) }\end{array}$ \\
\hline \multicolumn{5}{|c|}{ Análogos prandiales } \\
\hline Lispro & $\begin{array}{l}\text { Inversión de prolina en B28 y lisina en } \\
\text { B29 }\end{array}$ & $\leq 15 \mathrm{~min}$ & 30-90. & $4-5$ \\
\hline Aspart & $\begin{array}{l}\text { Cambio de prolina en B28 por ácido } \\
\text { aspártico }\end{array}$ & $\leq 15 \mathrm{~min}$ & $30-90$ & $4-5$ \\
\hline Glulisina & $\begin{array}{l}\text { Cambio de lisina en B29 por ácido } \\
\text { glutámico y aspargina en B3 por lisina }\end{array}$ & $10-15 \mathrm{~min}$ & $30-60$ & $4-5$ \\
\hline \multicolumn{5}{|c|}{ Análogos basales } \\
\hline Glargina & $\begin{array}{l}\text { Adición de } 2 \text { residuos de arginina al } \\
\text { final de la cadena B y reemplazo de } \\
\text { aspargina en A21 por glicina }\end{array}$ & $2-4 \mathrm{hr}$ & No presenta peak & $20-24$ \\
\hline Detemir & $\begin{array}{l}\text { Adición de ácido mirístico a lisina en } \\
\text { B29 y remoción de treonina en B30 }\end{array}$ & $\leq 15-30 \mathrm{~min}$ & No presenta peak & 12 \\
\hline
\end{tabular}

\section{Tabla 2. Estudios recientes con uso de análogos de insulinas ultrarrápidos}

\begin{tabular}{|c|c|c|c|c|}
\hline $\begin{array}{l}\text { Autor y año } \\
\text { publicación }\end{array}$ & Diseño & $\mathrm{N}$ & $\begin{array}{l}\text { Edad de los } \\
\text { pacientes } \\
\text { (años) }\end{array}$ & Conclusiones \\
\hline \multicolumn{5}{|l|}{ Lispro } \\
\hline $\begin{array}{l}\text { Anderson et al, } \\
1997(20)\end{array}$ & $\begin{array}{l}\text { Multicéntrico, } \\
\text { randomizado, abierto }\end{array}$ & 1008 & Adultos & $\begin{array}{l}\text { Lispro logra mejor control glicémico postprandial y } \\
\text { reduce las hipoglicemias vs insulina cristalina }\end{array}$ \\
\hline $\begin{array}{l}\text { Deeb et al, } \\
2001(25)\end{array}$ & Randomizado, prospectivo & 61 & 3-12 & $\begin{array}{l}\text { En niños prepuberales el uso de lispro preprandial } \\
\text { es seguro y mejora glicemias postprandiales } \\
\text { vs insulina cristalina }\end{array}$ \\
\hline $\begin{array}{l}\text { Chase et al, } \\
2001(26)\end{array}$ & Prospectivo & 884 & $0-<18$ & $\begin{array}{l}\text { Lispro reduce la HbA1 sin aumentar el nesgo de } \\
\text { hipoglicemia }\end{array}$ \\
\hline \multicolumn{5}{|l|}{ Aspart } \\
\hline $\begin{array}{l}\text { Home et al, } \\
1998 \text { (37) }\end{array}$ & $\begin{array}{l}\text { Multicéntrico, } \\
\text { randomizado, doble ciego, } \\
\text { con entrecruzamiento }\end{array}$ & 90 & $18-60$ & $\begin{array}{l}\text { Aspart mejora glicemias postprandiales vs insulina } \\
\text { cristalina. Menor incidencia de hiper e hipoglicemia }\end{array}$ \\
\hline $\begin{array}{l}\text { Mortensen et al, } \\
2000 \text { (34) }\end{array}$ & $\begin{array}{l}\text { Randomizado, doble ciego, } \\
\text { con entrecruzamiento }\end{array}$ & 9 & $6-12$ & $\begin{array}{l}\text { En niños al igual que en adultos, se observa un inicio } \\
\text { de acción más rápido de aspart vs insulina cristalina }\end{array}$ \\
\hline $\begin{array}{l}\text { Plank et al, } \\
2002 \text { (32) }\end{array}$ & $\begin{array}{l}\text { Randomizado, doble ciego, } \\
\text { con entrecruzamiento }\end{array}$ & 24 & $36 \pm 8$ & $\begin{array}{l}\text { Lispro y Aspart son igualmente efectivas en el control } \\
\text { de la hiperglicemia postprandial }\end{array}$ \\
\hline $\begin{array}{l}\text { Danne et al, } \\
2003 \text { (39) }\end{array}$ & Randomizado, prospectivo & 42 & $6-12$ & $\begin{array}{l}\text { Uso de aspart postprandial es segura y efectiva en niños } \\
\text { y adolescentes }\end{array}$ \\
\hline \multicolumn{5}{|l|}{ Glulisina } \\
\hline $\begin{array}{l}\text { Dailey et al, } \\
2004 \text { (43) }\end{array}$ & $\begin{array}{l}\text { Randomizado, } \\
\text { multicéntrico, abierto, en } \\
\text { grupos paralelos }\end{array}$ & 876 & $\geq 18$ & $\begin{array}{l}\text { Dos dosis de glulisina al día asociada a NPH mejora el } \\
\text { control metabólico vs insulina cristalina en DM2 }\end{array}$ \\
\hline
\end{tabular}




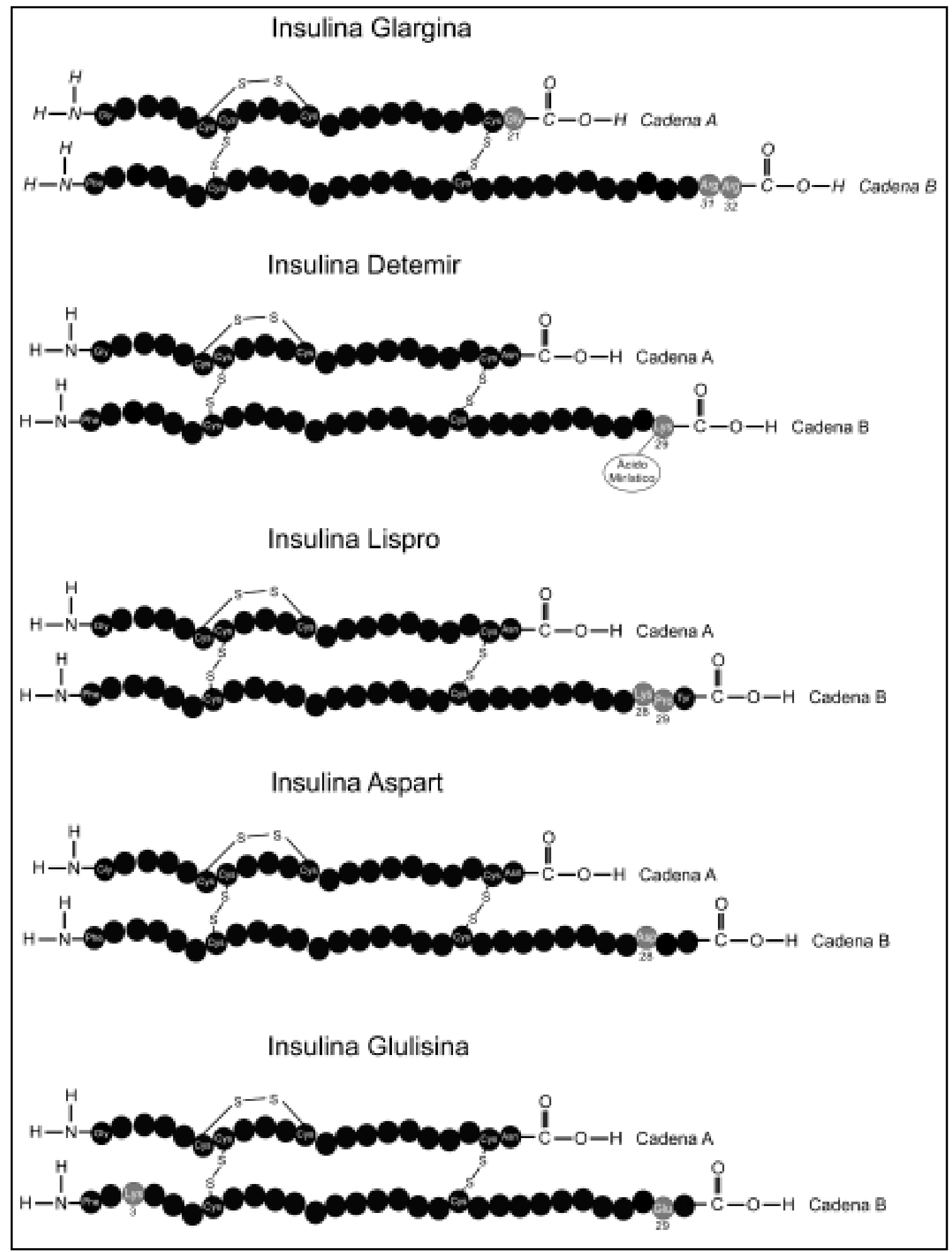

FIgURA 2. Cambios en la secuencia aminoacídica de los diferentes análogos de insulina. 
Los análogos de insulina conocidos como ultrarápidos disponibles actualmente son: lispro, aspart y glulisina, y se caracterizan por modificaciones en la cadena $B$, que evitan la disposición $ß$ plegada y con ello la formación de los hexámeros, tendiendo a permanecer como monómeros y dímeros en el subcutáneo, lo cual acelera su absorción ${ }^{15}$.

Estos cambios estructurales adelantaron el momento de máxima acción y permitieron lograr insulinemias máximas superiores a las alcanzadas con la IC. Este perfil farmacocinético permite, a diferencia de la cristalina, la inyección al momento de comer, flexibilizando el tratamiento. Gracias a su mecanismo de acción, los análogos son el tipo de insulina ideal para usar como suplemento o refuerzo en caso de comidas fuera del horario programado. Más aún, su rápida absorción permite el uso inmediatamente después de comer, facilitando el manejo en niños menores, en quienes es difícil estimar a priori la cantidad de alimentos que van a ingerir. Asimismo, el uso posterior a la comida permite ajustar la dosis de insulina según la ingesta ${ }^{5,6}$.

Otra similitud con la secreción fisiológica de insulina que presentan los análogos rápidos, es su menor tiempo total de acción, generalmente alrededor de $4 \mathrm{~h}$, por lo tanto, carecen de efecto basal y se comportan como verdaderas insulinas prandiales $^{14,16}$. Estas características reducen el riesgo de hipoglicemia y la necesidad de colaciones.

Insulina lispro. Fue el primer análogo en desarrollarse, a principios de la década 1990-99. Su nombre se debe a la inversión de los residuos prolina en posición B28 y lisina en B29 (Lys $\left.{ }^{\mathrm{B} 28} \mathrm{Pro}^{\mathrm{B} 29}\right)^{15}$ (Figura 2). Este cambio no altera su unión al receptor de insulina; su afinidad es semejante a la de la insulina humana, aumenta su unión al receptor de IGF-1 en 1,5 veces, pero corresponde sólo al $0,1 \%$ de la que presenta IGF-1 17 .

Este cambio estructural en la cadena B reduce 300 veces la dimerización de las cadenas proteicas ${ }^{18}$. Su acción se inicia en los primeros $15 \mathrm{~min}$ postinyección, alcanza un máximo a los 90 min, finalizando a las 4-5 $\mathrm{h}^{19}$. Logra niveles plasmáticos más altos durante el período postprandial, disminuyendo la hiperglicemia en éste ${ }^{20}$. Al comparar la farmacocinética de lispro con IC, su efecto máximo es más precoz. Howey, demostró que una inyección de $10 \mathrm{U}$ de lispro logra un efecto máximo a los 99\$39 versus
179 \pm 93 min de la $\mathrm{IC}^{19}$. Lispro respecto a cristalina presenta menor variabilidad intrasujeto y según el sitio anatómico inyectado ${ }^{19,21,22}$, siendo, por lo tanto, más predecible su efecto biológico. Además, el tiempo en alcanzar su máximo es independiente de la dosis administrada, a diferencia de la IC cuyo efecto máximo se incrementa a mayor dosis 23 .

Múltiples estudios controlados en niños y adultos han demostrado beneficios en el control metabólico comparada con $\mathrm{IC}^{24,25}$. Chase et al, en un seguimiento de niños y adolescentes DM1 observaron que lispro redujo significativamente la HbA1c en todos los grupos etáreos ${ }^{26}$. En algunos estudios, las diferencias con IC han sido sutiles, en parte debido a que se compara su efecto con IC administrada media hora antes de comer, hecho que es infrecuentemente cumplido por los pacientes fuera de un protocolo de investigación ${ }^{10}$.

Lispro disminuye los episodios de hipoglicemia versus IC $^{20}$. Este beneficio se debe a una absorción menos variable ${ }^{19,21,22}$, así como a su acción como verdadera insulina prandial. Anderson comparó su uso versus cristalina en pacientes tratados en forma randomizada durante 6 meses, y lispro redujo en $12 \%$ las hipoglicemias, principalmente nocturnas ${ }^{20}$.

Al igual que en DM1, en DM2, los análogos han demostrado beneficios en el manejo de la hiperglicemia postprandial ${ }^{27}$. En adultos con DM2 con mal control metabólico en tratamiento con gliburide, al asociar lispro con las comidas logró una mejoría del control metabólico superior que al agregar NPH o metformina ${ }^{27}$.

Los principales inconvenientes de esta insulina incluyen su mayor $\operatorname{costo}^{28} \mathrm{y}$ falta de efecto a las 4$6 \mathrm{~h}$, obligando frecuentemente a aumentar la dosis de insulina basal ${ }^{24,28}$. Aunque lispro no es idéntica a la insulina natural, no produce reacciones alérgicas, pero induce mayor síntesis de anticuerpos en pacientes sin insulinoterapia previa ${ }^{29}$.

Su uso no está aprobado en embarazadas, debido a su posible paso transplacentario y a evidencias de favorecer la progresión de la retinopatía ${ }^{30}$. Es posible que pronto sea autorizada, ya que recientemente se observó que lispro atraviesa la placenta sólo en caso de dosis extremadamente elevadas ${ }^{31}$.

Insulina aspart. De aparición posterior a lispro, en este análogo se sustituyó la prolina en posición B28 por ácido aspártico, lo cual evita la formación 
de hexámeros y acelera su paso a la circulación. Presenta un tiempo de inicio, máximo efecto y duración indistinguible de lispro ${ }^{32}$. Asimismo, presenta menor variabilidad intra e interindividual comparada con cristalina 33,34 .

Al igual que lispro, su rápida absorción desde el subcutáneo permite su uso como insulina prandial. Clínicamente, este perfil farmacocinético determina glicemias postprandiales inferiores que con $\mathrm{IC}^{33}$, permite administrarla después de comer y ajustar la dosis según la ingesta ${ }^{34}$.

Los beneficios de aspart se traducen en mejorar la calidad de vida $32,35,36$, disminución de las hipoglicemias ${ }^{37,38}$ y la $\mathrm{HbA1c}^{39}$. Induce menos reacciones inmunológicas versus NPH, IC y lispro, transformándose en una opción segura de tratamiento ${ }^{40}$. Sin embargo, aún no está autorizado su uso en embarazadas.

En DM2, se ha introducido aspart asociada a hipoglicemiantes orales (metformina, rosiglitazona y glimepiride), logrando disminuir significativamente la HbA1c a largo plazo ${ }^{41}$.

Glulisina. Este análogo ultrarrápido comenzó su comercialización en 2004 (Apidra ${ }^{\circledR}$, Laboratorio Aventis). Al igual que los otros, presenta modificaciones estructurales; cambio de lisina en B29 por ácido glutámico y reemplazo de aspargina en B3 por lisina ${ }^{42}$.

Existen escasos estudios con glulisina en DM1 y DM2 ${ }^{43}$. En adultos, el uso de glulisina y lispro 15 minutos preprandial asociado a glargina como basal, demostró un control metabólico y episodios de hipoglicemia equivalentes ${ }^{44}$. Otro estudio con IC precomida o glulisina 0-15 minutos preprandial o 0-20 minutos desde el inicio de la comida, asociado a glargina, demostró un descenso superior de la HbA1c en los pacientes con glulisina ${ }^{42}$.

No está estudiado su efecto en embarazadas ni en niños, proscribiéndose su uso en ellos actualmente. Se han descrito reacciones alérgicas locales como eritema, dolor y edema, con escasos reportes de reacciones sistémicas graves ${ }^{42}$.

\section{FISIOLOGÍA DE LA SECRECIÓN DE INSULINA DURANTE EL PERÍODO INTERPRANDIAL}

Paradojalmente, la insulina también cumple un rol durante el período de ayuno (insulina basal»), encon- trándose en bajas concentraciones sanguíneas. Durante este período inhibe la neoglucogénesis hepática, evitando la hiperglicemia de ayuno. Asimismo, inhibe la lipólisis desde el tejido adiposo, disminuyendo el aporte de ácidos grasos al hígado y con ello, la cetogénesis ${ }^{28}$. Por lo tanto, ante el déficit de insulina basal se produce hiperglicemia de ayuno y cetosis.

La secreción basal de insulina al sistema portal es continua, en niveles muy bajos, alcanzando directamente el hígado donde ejerce su principal efecto. Pese a que ésta es continua durante el período interprandial, los niveles varían durante el día; por ejemplo, los requerimientos de insulina basal son 30\% mayores en la madrugada que en las primeras horas de la noche ${ }^{45}$. El aporte exógeno de insulina en sujetos con DM1 no se libera directamente a la circulación portal y tampoco imita las variaciones en los niveles de insulina basal como ocurre en el sujeto sano.

Reemplazo de insulina durante el período interprandial o basal

Insulina NPH. Esta insulina contiene protamina, proteína básica extraída de la esperma de los peces, y zinc en proporciones estequiométricas $^{46,47}$. Considerada una insulina de acción intermedia, inicia su acción a las $2 \mathrm{~h}$ y tiene una duración total del efecto entre 13-18 h, con una acción máxima a las 5-8 $\mathrm{h}^{48,49}$.

Su uso como insulina basal tiene varias limitaciones. En primer lugar, tiene un perfil farmacocinético con un máximo, alcanzando insulinemias que se asemejan a una insulina prandial. Este perfil de acción mezcla elementos de insulina basal y prandial, obligando al paciente a cubrir el período de máxima acción con comidas a horarios fijos y creando problemas en el control glicémico nocturno. La inyección vespertina de NPH tiene su máximo efecto en las primeras horas de la madrugada, cuando aún no ha comenzado el fenómeno del amanecer, siendo causa frecuente de hipoglicemia nocturna ${ }^{45,50}$.

Otra desventaja de la NPH son las variaciones de su efecto de hasta 40-50\% intrasujeto, reflejando una respuesta clínicamente impredecible ${ }^{51,52}$. Esta variabilidad se debe, en parte, a que la NPH debe ser homogeneizada, moviendo suavemente el frasco antes de inyectarse, acción que no realizan siempre los pacientes. Además, el momento de su máximo efecto es también altamente 
Tabla 3. Estudios recientes con uso de análogos de insulinas para uso como aporte basal

\begin{tabular}{|c|c|c|c|c|}
\hline $\begin{array}{l}\text { Autor y año } \\
\text { publicación }\end{array}$ & Diseño & $\mathrm{N}$ & $\begin{array}{l}\text { Edad de los } \\
\text { pacientes } \\
\text { (años) }\end{array}$ & Conclusiones \\
\hline \multicolumn{5}{|l|}{ Glargina } \\
\hline $\begin{array}{l}\text { Lepore et al, } \\
2000 \text { (49) }\end{array}$ & $\begin{array}{l}\text { Randomizado, secuencial y } \\
\text { con entrecruzamiento }\end{array}$ & 20 & $32 \pm 2$ & $\begin{array}{l}\text { Glargina carece de peak y tiene menor } \\
\text { variabilidad intersujeto que NPH y ultralenta }\end{array}$ \\
\hline $\begin{array}{l}\text { Ratner et al, } \\
2000(60)\end{array}$ & $\begin{array}{l}\text { Randomizado, multicéntrico, } \\
\text { en grupos paralelos }\end{array}$ & 534 & $38,5 \pm 12,04$ & $\begin{array}{l}\text { Con glargina los pacientes presentan menos } \\
\text { hipoglicemia y menores glicemias de ayuno } \\
\text { vs NPH en } 1 \text { ó } 2 \text { dosis diarias }\end{array}$ \\
\hline $\begin{array}{l}\text { Chase et al, } \\
2003 \text { (58) }\end{array}$ & Prospectivo & 114 & $2-18$ & $\begin{array}{l}\text { Glargina reduce los episodios de hipoglicemia en } \\
\text { niños y adolescentes con control metabólico subóptimo }\end{array}$ \\
\hline $\begin{array}{l}\text { Kaplan et al, } \\
2004 \text { (54) }\end{array}$ & $\begin{array}{l}\text { Prospectivo, randomizado, } \\
\text { con entrecruzamiento }\end{array}$ & 14 & $13,5 \pm 0,5$ & $\begin{array}{l}\text { La mezcla de lispro o aspart en la misma jeringa no } \\
\text { deteriora el control metabólico y no tiene efectos } \\
\text { adversos }\end{array}$ \\
\hline \multicolumn{5}{|l|}{ Detemir } \\
\hline $\begin{array}{l}\text { Danne et al, } \\
2003 \text { (52) }\end{array}$ & $\begin{array}{l}\text { Randomizado, abierto, con } \\
\text { entrecruzamiento }\end{array}$ & 34 & Jun-65 & $\begin{array}{l}\text { Detemir tiene una respuesta más predecible vs } \\
\mathrm{NPH} \text {, menor variabilidad y efecto semejante entre } \\
\text { las diferentes edades }\end{array}$ \\
\hline $\begin{array}{l}\text { Vague et al, } \\
2003 \text { (65) }\end{array}$ & $\begin{array}{l}\text { Prospectivo, en grupos } \\
\text { paralelos, abierto }\end{array}$ & 448 & $\begin{array}{c}38,9 \pm 13,3 \\
\text { y } 41,8 \pm 12,2\end{array}$ & $\begin{array}{l}\text { Detemir tiene un efecto más predecible, se } \\
\text { asocia a menor número de hipoglicemias y a un } \\
\text { menor peso en sus usuarios vs NPH }\end{array}$ \\
\hline $\begin{array}{l}\text { Heise et al, } \\
2004 \text { (51) }\end{array}$ & Randomizado, doble ciego & 32 & $38 \pm 10$ & $\begin{array}{l}\text { Detemir tiene menor variablidad intersujetos, y } \\
\text { efecto más predecible que NPH y glargina }\end{array}$ \\
\hline
\end{tabular}

variable. Danne demostró que NPH produce un máximo efecto más temprano a edades menores $^{52}$; a las $3 \mathrm{~h}$ en niños, a las $6 \mathrm{~h}$ en adolescentes $\mathrm{y}$ a las $8 \mathrm{~h}$ en adultos.

Análogos basales de insulina (Tablas 1 y 3 y Figuras 1 y 2). Estos análogos se han diseñado con el objetivo de obtener insulinas de acción prolongada con un perfil farmacocinético plano $\mathrm{y}$, por ende, más semejantes a la insulina basal. Se encuentran disponibles en el mercado glargina y detemir.

Insulina glargina (HOE901). Fue el primer análogo basal desarrollado, a la venta en Estados Unidos de Norteamérica (EEUU) y Europa desde el año 2000. Esta insulina es el resultado de dos modificaciones estructurales; elongación de la cadena B en su porción final con 2 residuos de arginina, que modifican su punto isoeléctrico y la hacen soluble a pH ácido $\mathrm{y}$, por ende, menos soluble a pH fisiológico. La segunda modificación consistió en el reemplazo de la aspargina en posición A21 por glicina, confiriéndole mayor estabilidad. Su presentación es líquida en una solución ácida e incolora, que al contactarse con el pH neutro del subcutáneo precipita. El precipitado se disocia lentamente a hexámeros y posteriormente a dímeros y monómeros, dando una absorción sostenida y lenta que determina niveles plasmáticos planos ${ }^{53}$.

El hecho de ser una solución ácida obliga a ser inyectada separada de la insulina prandial, aumentando el número de inyecciones totales diarias $^{6}$. A pesar de esto, recientemente, se publicó un reporte del uso de glargina mezclada con lispro o aspart en la misma jeringa, sin presentar deterioro del control glicémico ni aumento de las hipoglicemias ${ }^{54}$. Glargina tiene la ventaja de ser 
una solución líquida que no requiere ser homogeneizada.

$\mathrm{Su}$ inicio de acción se produce a las 2-4 h, manteniendo niveles estables por más de 24 $\mathrm{h}^{49,55}$. Este perfil farmacodinámico permite su administración en una sola inyección diaria, a cualquier hora del día, para otorgar una insulinemia basal, asociada a análogos ultrarrápidos con las comidas ${ }^{56}$. Ocasionalmente, especialmente en niños menores, se requieren dos dosis diarias.

El cambio del tratamiento desde insulina NPH a glargina permite una disminución de la dosis de insulina basal en 20-30\%. En cambio, las dosis de insulina prandial, en general, no requieren grandes modificaciones ${ }^{57}$.

Glargina se ha asociado, en niños y adultos, a menores episodios de hipoglicemia ${ }^{58-60}$, especialmente con la administración matinal ${ }^{56}$. Su uso ha logrado una mejoría en el control metabólico en DM1 y DM2 comparado con $\mathrm{NPH}^{61}$; incluso consigue un control glicémico semejante a la bomba de infusión de insulina ${ }^{62}$.

El uso de glargina no ha sido aún aprobado en menores de 6 años, pero estudios han evidenciado beneficios en este grupo, incluso en lactantes $^{63}$. Uno de los inconvenientes de glargina, en este grupo etáreo, es la restricción en la ingesta de colaciones a deshora, hecho que puede superarse utilizando una dosis nocturna de glangina asociada a NPH matinal ${ }^{58}$. Su administración no está permitida aún en embarazadas ni en pacientes con insuficiencia hepática o renal ${ }^{53}$.

Insulina detemir. Es el análogo de acción prolongada [Lys ${ }^{\mathrm{B} 29}\left(\mathrm{~N}^{\mathrm{E}}\right.$-tetracanoyl)des(B30)] de más reciente desarrollo. Su efecto prolongado se produce por un mecanismo diferente a los otros análogos. La adición de un ácido graso de C14 (ácido mirístico) a la lisina en B29 le confiere capacidad de unirse a albúmina y de esta forma, actúa como un reservorio de insulina, prolongando su efecto. La remoción de una treonina en B30 facilita la formación de hexámeros, alargando su acción. Esta proteína es soluble a $\mathrm{pH}$ neutro y alcanza su efecto a los 90 min de la administración, con niveles más bien planos, permitiendo su uso como insulina basal ${ }^{51,52,64}$.

Detemir generalmente debe ser usada en dos inyecciones diarias y es de alto costo. Su principal ventaja está dada porque es la insulina basal con la menor variabilidad intrasujeto entre las dosis $^{51,52,65}$. Comparada con $\mathrm{NPH}$, tiene menos variaciones en su efecto en el mismo sujeto, su tiempo de máximo efecto no cambia según la edad y logra un mejor control metabólico $51,52,65$. $\mathrm{Al}$ igual que glargina, existe menor riesgo de hipoglicemia diurna y nocturna ${ }^{65}$.

Tratamiento intensificado con análogos de insulina (Tabla 1 y Figura 1).

$\mathrm{Su}$ objetivo es imitar la secreción natural de insulina, para lo cual se utiliza una insulina basal, asociada a una de acción rápida en cada una de las comidas principales, esquema conocido como bolo-basal ${ }^{66}$. Como basal se ha utilizado $\mathrm{NPH}$, ultralenta, glangina o detemir y como prandial, el paciente puede recibir IC, lispro, aspart o glulisina.

Se han publicado diversos esquemas intensificados con múltiples dosis de NPH, entre 2 y 4 dosis diarias, pero éstos han mostrado un peor control metabólico y mayor complejidad que el tratamiento con glargina ${ }^{61,67}$. El año 2003 se publicó el primer esquema intensificado en DM1 realizado en un hospital público chileno. Consistió en el uso de dos dosis de NPH y tres dosis diarias de cristalina, lográndose un control metabólico semejante a otros centros que tratan adolescentes en forma intensificada ${ }^{5}$. Posteriormente, este mismo grupo del Hospital San Borja Arriarán, que no cuenta con glargina, publicó sus resultados con tres dosis diarias de $\mathrm{NPH}^{6}$. Este esquema fue el preferido en adolescentes, permitiendo mayor flexibilidad en el horario de la cena y aumentar la ingesta de la colación en la tarde.

En el caso de usar glargina o detemir asociada a análogos ultrarrápidos, se separa el aporte de insulina basal de la prandial. Este esquema elimina la necesidad de colaciones, otorga mayor libertad y flexibilidad en los horarios de alimentación y en la cantidad de carbohidratos por comida ${ }^{66,68}$. Si el paciente sabe cuantificar los carbohidratos de la comida a ingerir y conoce la cantidad de ellos cubiertos por una unidad de insulina, puede modificar la dosis de insulina prandial según la ingesta y la glicemia ${ }^{6,69}$.

Otra forma de realizar tratamiento intensificado es con bomba de infusión continua subcutánea de insulina. Este tipo de tratamiento permite el aporte permanente de insulina con velocidad 
variable, permitiendo ajustes en el aporte de insulina basal y prandial. El uso de bomba con análogos de insulina ultrarrápidos, por su absorción rápida, logra que la bomba subcutánea se asemeje a una infusión endovenosa a la vía sistémica $^{70}$.

\section{REFERENCIAS}

1. The Diabetes Control and Complcations Trial ReSEARCH GROUP. The effect of intensive treatment of diabetes on the development and progression of long-term complications in insulin-dependent diabetes mellitus. The Diabetes Control and Complications Trial Research Group. N Engl J Med 1993; 329: 977-86.

2. UK Prospective Diabetes Study (UKPDS) Group. Intensive blood-glucose control with sulphonylureas or insulin compared with conventional treatment and risk of complications in patients with type 2 diabetes (UKPDS 33). Lancet 1998; 352: 837-53.

3. HIRSCH IB. Intensive treatment of type 1 diabetes. Med Clin North Am 1998; 82: 689-719.

4. Aylwin C, Codner E, García H, Pérez J, Ugarte F, Escobar M ET aL. Protocolo AUGE. Diabetes Mellitus tipo 1. Santiago: Ministerio de Salud de Chile; 2004.

5. Codner E, Merice V, García H, López C, Cáceres J, GAETE X ET AL. Resultados de un programa multidisciplinario de tratamiento intensificado de la Diabetes Mellitus tipo 1 (DM1) en un Hospital Público. Rev Méd Chile 2003; 131: 857-64.

6. Codner E, Merice MV, Román R, HrLic I, Martínez A, Unanue $\mathrm{N}$ et al. Nuevos esquemas de tratamiento con insulina en niños y adolescentes con Diabetes Mellitus tipo 1 (DM1) en un Hospital Público. Rev Chil Pediatr 2004; 75: 520-9.

7. Bow GB, Dimitriadis GD, Pehling GB, Baker BA, HAYMond MW, CRYeR PE et al. Abnormal glucose counterregulation after subcutaneous insulin in insulin-dependent diabetes mellitus. N Engl J Med 1984; 310: 1706-11.

8. Ciofetta M, Lawi C, Del Sindaco P, Torlone E, Pampaneli S, Mauro L et al. Contribution of postprandial versus interprandial blood glucose
En conclusión, los análogos de insulina han permitido ofrecer nuevas alternativas terapéuticas a los pacientes con diabetes mellitus, pero aún no se logra homologar la secreción fisiológica de insulina.

to HbA1c in type 1 diabetes on physiologic intensive therapy with lispro insulin at mealtime. Diabetes Care 1999; 22: 795-800.

9. Burge MR, Schade DS. Insulins. Endocrinol Metab Clin North Am 1997; 26: 575-98.

10. Del Sindaco P, Ciofetta M, Law C, Perrielo G, Pampanem S, Torlone E et al. Use of the shortacting insulin analogue lispro in intensive treatment of type 1 diabetes mellitus: importance of appropriate replacement of basal insulin and time-interval injection-meal. Diabet Med 1998; 15: 592-600.

11. Dimitriadis GD, Gerich JE. Importance of timing of preprandial subcutaneous insulin administration in the management of diabetes mellitus. Diabetes Care 1983; 6: 374-7.

12. Heinemann L, Richter B. Clinical pharmacology of human insulin. Diabetes Care 1993; 16 Suppl 3: 90-100.

13. Heinemann L, Chantelau EA, Starke AA. Pharmacokinetics and pharmacodynamics of subcutaneously administered U40 and U100 formulations of regular human insulin. Diabete Metab 1992; 18: 21-4.

14. StANDL E. Insulin analogues - state of the art. Horm Res 2002; 57 Suppl 1: 40-5.

15. Holeman F, Hoekstra JB. Insulin lispro. N Engl J Med 1997; 337: 176-83.

16. WILAMs RM, Dunger DB. Insulin treatment in children and adolescents. Acta Paediatr 2004; 93: 440-6.

17. Slieker LJ, Brooke GS, Chance RE, Al E. Insulin and IGF-1 analogs: novel approaches to improved insulin pharmacokinetics. En: Le Roith D, M.K. R, eds. Current directions in insulin-like growth factor research. New York: Plenum Press, 1994; 25-32.

18. Ciszak E, Beals JM, Frank BH, Baker JC, Carter ND, Sмттн GD. Role of C-terminal B-chain residues in 
insulin assembly: the structure of hexameric LysB28ProB29-human insulin. Structure 1995; 3: 615-22.

19. Howey DC, Bowsher RR, Bruneue RL, Woodworth JR. [Lys(B28), Pro(B29)-human insulin. A rapidly absorbed analogue of human insulin. Diabetes 1994; 43: 396-402.

20. Anderson JH Jr, Brunewe RL, Koivisto VA, Pfutzner A, Trautmann ME, Vignati L et al. Reduction of postprandial hyperglycemia and frequency of hypoglycemia in IDDM patients on insulin-analog treatment. Multicenter Insulin Lispro Study Group. Diabetes 1997; 46: 265-70.

21. Ter Braak EW, Woodworth JR, Bianchi R, Cerimele B, ERKelens DW, ThijSSEN JH et al. Injection site effects on the pharmacokinetics and glucodynamics of insulin lispro and regular insulin. Diabetes Care 1996; 19: 1437-40.

22. Heinemann L, Heise T, Wahl LC, Trautmann ME, Ampudia J, Starke AA et al. Prandial glycaemia after a carbohydrate-rich meal in type I diabetic patients: using the rapid acting insulin analogue [Lys(B28), Pro(B29)] human insulin. Diabet Med 1996; 13: 625-9.

23. Tuominen JA, Karonen SL, Melamies L, Bow G, KorvisTo VA. Exercise-induced hypoglycaemia in IDDM patients treated with a short-acting insulin analogue. Diabetologia 1995; 38: 106-11.

24. Ebeung P, Jansson PA, Smith U, Laш C, Bош GB, KoIvISTo VA. Strategies toward improved control during insulin lispro therapy in IDDM. Importance of basal insulin. Diabetes Care 1997; 20: 1287-9.

25. Deeb LC, Holcombe JH, Brunelue R, Zalani S, Brink S, JeNNER M ET aL. Insulin lispro lowers postprandial glucose in prepubertal children with diabetes. Pediatrics 2001; 108: 1175-9.

26. Chase HP, Lockspeiser T, Peery B, Shepherd M, Mackenzie T, Anderson J et al. The impact of the diabetes control and complications trial and humalog insulin on glycohemoglobin levels and severe hypoglycemia in type 1 diabetes. Diabetes Care 2001; 24: 430-4.

27. Bastyr EJ $3^{\text {RD }}$, Stuart CA, Brodows RG, Schwartz S, GraF CJ, Zagar A ET aL. Therapy focused on lowering postprandial glucose, not fasting glucose, may be superior for lowering HbA1c. IOEZ Study Group. Diabetes Care 2000; 23: 1236-41.

28. Вош GB. Physiological insulin replacement in type 1 diabetes mellitus. Exp Clin Endocrinol Diabetes 2001; 109 Suppl 2: S317-332.
29. Fineberg NS, Fineberg SE, ANDerson JH, Birkett MA, GIBSON RG, HuFFERD S. Immunologic effects of insulin lispro [Lys (B28), Pro (B29) human insulin] in IDDM and NIDDM patients previously treated with insulin. Diabetes 1996; 45: 1750-4.

30. LOUKOVAara S, Immonen I, Teramo KA, KaAja R. Progression of retinopathy during pregnancy in type 1 diabetic women treated with insulin lispro. Diabetes Care 2003; 26: 1193-8.

31. Boskovic R, Feig DS, Derewlany L, Knie B, Portnoi G, Koren G. Transfer of insulin lispro across the human placenta: in vitro perfusion studies. Diabetes Care 2003; 26: 1390-4.

32. Plank J, Wutte A, Brunner G, Siebenhofer A, SemLtsch B, Sommer R et al. A direct comparison of insulin aspart and insulin lispro in patients with type 1 diabetes. Diabetes Care 2002; 25: 2053-7.

33. Heinemann L, Weyer C, Rauhaus M, Heinrichs S, HeISE T. Variability of the metabolic effect of soluble insulin and the rapid-acting insulin analog insulin aspart. Diabetes Care 1998; 21: 1910-4.

34. Mortensen HB, Lindholm A, Olsen BS, Hy山eberg B. Rapid appearance and onset of action of insulin aspart in paediatric subjects with type 1 diabetes. Eur J Pediatr 2000; 159: 483-8.

35. Devries JH, Lundholm A, Jacobsen JL, Heine RJ, Home PD. A randomized trial of insulin aspart with intensified basal NPH insulin supplementation in people with Type 1 diabetes. Diabet Med 2003; 20: 312-8.

36. REyNolds NA, WagSTAFF AJ. Insulin Aspart: A Review of its Use in the Management of Type 1 or 2 Diabetes Mellitus. Drugs 2004; 64: 1957-74.

37. Home PD, Lindholm A, Hyueberg B, Round P. Improved glycemic control with insulin aspart: a multicenter randomized double-blind crossover trial in type 1 diabetic patients. UK Insulin Aspart Study Group. Diabetes Care 1998; 21: 1904-9.

38. Hermansen K, Fontaine P, Kukolja KK, Peterkova V, Leth G, GaLl MA. Insulin analogues (insulin detemir and insulin aspart) versus traditional human insulins (NPH insulin and regular human insulin) in basal-bolus therapy for patients with type 1 diabetes. Diabetologia 2004; 47: 622-9.

39. Danne T, Aman J, Schober E, Deiss D, Jacobsen JL, FRIBERG HH ET AL. A comparison of postprandial and preprandial administration of insulin aspart in children and adolescents with type 1 diabetes. Diabetes Care 2003; 26: 2359-64. 
40. Yasuda H, Nagata M, Moriyama $H$, FujihiRa $K$, Kotani R, Yamada K et al. Human insulin analog insulin aspart does not cause insulin allergy. Diabetes Care 2001; 24: 2008-9.

41. Poulsen MK, Henriksen JE, Hother-Nielsen O, BeckNIELSEN H. The combined effect of triple therapy with rosiglitazone, metformin, and insulin aspart in type 2 diabetic patients. Diabetes Care 2003; 26: 3273-9.

42. BARLOCCO D. Insulin glulisine. Aventis Pharma. Curr Opin Investig Drugs 2003; 4: 1240-4.

43. Dailey G, Rosenstock J, Moses RG, Ways K. Insulin glulisine provides improved glycemic control in patients with type 2 diabetes. Diabetes Care 2004; 27: 2363-8.

44. Aventis Pharmaceuticals Inc. Apidra (insulin glulisine [rDNA origin] injection). Rev April 2004a.

45. Perriello G, De Feo P, Torlone E, Fanelu C, Santeusanio F, Brunetti $\mathrm{P}$ et al. The dawn phenomenon in type 1 (insulin-dependent) diabetes mellitus: magnitude, frequency, variability, and dependency on glucose counterregulation and insulin sensitivity. Diabetologia 1991; 34: 21-8.

46. ScotT S, Fisher A. Studies on insulin with protamine. J Pharmacol Exp Ther 1936; 58.

47. Halas-Moler K, Jersidd M, Petersen K, SchlichtKru$\amalg \mathrm{J}$. Clinical studies on new insulin preparations with prolonged action; one daily injection with insulin zinc preparations. Ugeskr Laeger 1951; 113: 1767-71.

48. Starke AA, Heinemann L, Hohmann A, Berger M. The action profiles of human NPH insulin preparations. Diabet Med 1989; 6: 239-44.

49. Lepore M, Pampanelu S, Fanem C, Porcellati F, Bartocci L, Di Vincenzo A et al. Pharmacokinetics and pharmacodynamics of subcutaneous injection of long-acting human insulin analog glargine, NPH insulin, and ultralente human insulin and continuous subcutaneous infusion of insulin lispro. Diabetes 2000; 49: 2142-8.

50. Bow GB, Perriello G, Fanem CG, De Feo P. Nocturnal blood glucose control in type I diabetes mellitus. Diabetes Care 1993; 16 Suppl 3: 7189.

51. Heise T, Nosek L, Ronn BB, Endahl L, Heinemann L, KAPITZA C ET AL. Lower within-subject variability of insulin detemir in comparison to NPH insulin and insulin glargine in people with type 1 diabetes. Diabetes 2004; 53: 1614-20.
52. Danne T, Lupke K, Walte K, Von Schuetz W, GaL MA. Insulin detemir is characterized by a consistent pharmacokinetic profile across age-groups in children, adolescents, and adults with type 1 diabetes. Diabetes Care 2003; 26: 3087-92.

53. Giшes PS, FiggitT DP, Lamb HM. Insulin glargine. Drugs 2000; 59: 253-60.

54. Kaplan W, Rodríguez LM, Smith OE, Haymond MW, HEPTULA RA. Effects of mixing glargine and shortacting insulin analogs on glucose control. Diabetes Care 2004; 27: 2739-40.

55. Heinemann L, LnNeschova R, Rave $\mathrm{K}$, Hompesch B, SediaK M, Heise T. Time-action profile of the longacting insulin analog insulin glargine (HOE901) in comparison with those of NPH insulin and placebo. Diabetes Care 2000; 23: 644-9.

56. Hamann A, Matthaei S, Rosak C, Silvestre L A randomized clinical trial comparing breakfast, dinner, or bedtime administration of insulin glargine in patients with type 1 diabetes. Diabetes Care 2003; 26: 1738-44.

57. TAN CY, M Wilson D, Buckingham B. Initiation of insulin glargine in children and adolescents with type 1 diabetes. Pediatr Diabetes 2004; 5: 80-6.

58. Chase HP, Dixon B, Pearson J, Fiallo-Scharer R, WalRavens P, Kungensmith G et al. Reduced hypoglycemic episodes and improved glycemic control in children with type 1 diabetes using insulin glargine and neutral protamine Hagedorn insulin. J Pediatr 2003; 143: 737-40.

59. Murphy NP, Keane SM, Ong KK, Ford-Adams M, EDGe JA, ACERINI CL ET AL. Randomized cross-over trial of insulin glargine plus lispro or NPH insulin plus regular human insulin in adolescents with type 1 diabetes on intensive insulin regimens. Diabetes Care 2003; 26: 799-804.

60. Ratner R, Hirsch I, Neifing J, Garg S, Mecca T, WILson C. Less hypoglycemia with insulin glargine in intensive insulin therapy for type 1 diabetes. U.S. Study Group of Insulin Glargine in Type 1 Diabetes. Diabetes Care 2000; 23: 639-43.

61. Rossetti P, Pampanew S, Fanew C, Porcelati F, COSTA E, TORLONE E ET AL. Intensive replacement of basal insulin in patients with type 1 diabetes given rapid-acting insulin analog at mealtime: a 3-month comparison between administration of $\mathrm{NPH}$ insulin four times daily and glargine insulin at dinner or bedtime. Diabetes Care 2003; 26: 1490-6. 
62. Weintrob N, Benzaquen H, Galatzer A, Shautin S, LAZAR L, FAYMAN G ET AL. Comparison of continuous subcutaneous insulin infusion and multiple daily injection regimens in children with type 1 diabetes: a randomized open crossover trial. Pediatrics 2003; 112: 559-64.

63. Goonetileke R, Powtzzer M, Mann N. Insulin for toddlers with difficult diabetes. Diabetes Care 2004; 27: 1505.

64. Heinemann L, Sinha K, Weyer C, Loftager M, Hirschberger S, Heise T. Time-action profile of the soluble, fatty acid acylated, long-acting insulin analogue NN304. Diabet Med 1999; 16: 332-8.

65. Vague P, Seiam JL, Skeie S, De Lfeuw I, Elte JW, Haahr $\mathrm{H}$ ET AL Insulin detemir is associated with more predictable glycemic control and reduced risk of hypoglycemia than NPH insulin in patients with type 1 diabetes on a basal-bolus regimen with premeal insulin aspart. Diabetes Care 2003; 26: 590-6.

66. Hirsch IB, Farkas-Hirsch R, Skyler JS. Intensive insulin therapy for treatment of type I diabetes. Diabetes Care 1990; 13: 1265-83.
67. Lawi C, Ciofetta M, Del Sindaco P, Torlone E, Pampaneli S, Compagnucci P ET aL. Long-term intensive treatment of type 1 diabetes with the short-acting insulin analog lispro in variable combination with NPH insulin at mealtime. Diabetes Care 1999; 22: 468-77.

68. Franz MJ, Bantle JP, Beebe CA, Brunzell JD, Chiasson J-L, Garg A ET al. Evidence-Based Nutrition Principles and Recommendations for the Treatment and Prevention of Diabetes and Related Complications. Diabetes Care 2002; 25: 14898.

69. Rabasa-Lhoret R, Garon J, Langelier H, Poisson D, CHIASSON JL Effects of meal carbohydrate content on insulin requirements in type 1 diabetic patients treated intensively with the basal-bolus (ultralente-regular) insulin regimen. Diabetes Care 1999; 22: 667-73.

70. Weissberg-Benchell J, Antisdel-Lomaglio J, Seshadri R. Insulin pump therapy: a meta-analysis. Diabetes Care 2003; 26: 1079-87. 